\title{
Environmental Effects on Photomultiplication Propagation in Silicon
}

\author{
Matthew W. Fishburn and Edoardo Charbon \\ Delft University of Technology, Mekelweg 4, 2628 CD Delft, Netherlands \\ phone: +31-15-278-3667, fax: +31-15-278-6190, email: m.w.fishburn@tudelft.nl
}

Within the past decade, there has been a growing interest in multi-modality systems utilizing both position emission tomography (PET) and magnetic resonance imaging (MRI) techniques[1]. Much of this interest is focused on small animal imaging, with the soft-tissue contrast of MRI compensating for the poor contrast in PET[2]. However, due to the magnitude of magnetic fields inherent in small animal MRI systems, sensor imperviousness to strong magnetic fields is critical. To overcome this constraint, Geigermode avalanche photodiodes (GAPDs, also known as single-photon avalanche diodes or SPADs), which have been shown to be nearly impervious to magnetic fields as strong as 7T, are often proposed in PET-MRI systems[3]. While the timing characteristics of GAPDs under strong magnetic fields have been studied, the magnetic field's effect on avalanche propagation itself has not been examined in great detail. Understanding this relationship is critical to predicting at what magnetic field strength distortions to timing jitter may be seen, and also in understanding the relationship between the diode geometry, magnetic field, and observed jitter.

In this work we present measurements of the distortions to avalanche propagation under various magnetic fields and temperatures, observed with the use of position-sensitive GAPDs. Diodes that are long and thin have different avalanche quench times depending on the avalanche seed location, an effect caused by the avalanche propagating across the diode geometry, and sampling the quenching waveform's rise time allows an estimation of the avalanche propagation speed[4]. Additionally, characterization results from a shortrange, 18ps time-to-digital converter (TDC) in a strong magnetic field are presented.

On a CMOS chip, pill-shaped GAPDs with $6 \times 24 \mu \mathrm{m}^{2}$ bodies capped by two semi-circles of diameter $6 \mu \mathrm{m}$, which have a geometry suitable for observation of differing quench times depending on the avalanche seed position, are coupled to an 18ps, Vernier-delayline TDC via two comparators. The pill-shaped diodes are either completely open, completely covered, covered except for a $2 \mu \mathrm{m}$ opening in the diode's middle, or covered except for a $2 \mu \mathrm{m}$ opening on the edge of the diode's major axis. Use of these coverings confirmed that, due to the finite propagation speed, the avalanche quench time varies as a function of the seed position. Also included on the chip are circular GAPDs with a $12 \mu \mathrm{m}$ diameter. Bias signals allow compensation for TDC resolution changes from process, voltage and temperature variations. The CMOS chip is assembled on a daughterboard with a commercial, 60ps TDC for a time-offlight (TOF) measurement using a pulsed laser. A readout system based on a field-programmable gate array (FPGA) interfaces the chip to a computer workstation. A simplified diagram of the system is shown in Fig. 1.

A TDC density test[5] using a periodic stop input from the FPGA and a start signal from the dark counts of a GAPD was used to measure the on-chip TDC's resolution, differential nonlinearity, and integral nonlinearity. An external bias signal was used to trim the TDC for measurements at different temperatures, but was kept constant during measurements with a varying magnetic field. The TDC's resolution shows a statistically insignificant difference of $0.4 \mathrm{ps}$ between being under either a $<0.1 \mathrm{~T}$ or a 9.4T magnetic field. The commercial TDC compensates for distortions on-chip using measurements of an external clock signal; no significant resolution variation under a strong magnetic field was observed from this TDC following its internal compensation.

During all measurements, the breakdown voltage $\mathrm{V}_{\text {bd }}$ was determined by keeping the low comparator at a constant threshold and increasing the voltage applied to the GAPD until pulses appeared at the thresholder's output. $\mathrm{V}_{\text {bd }}$ is this applied voltage minus the threshold voltage. At room temperature, the breakdown voltage of the pill-shaped GAPDs was measured to be within $20 \mathrm{mV}$ of $18.6 \mathrm{~V}$ under a $9.4 \mathrm{~T}$ field and a $<0.1 \mathrm{~T}$ field. Over a range of temperatures from $-20^{\circ} \mathrm{C}$ to $50^{\circ} \mathrm{C}$, the breakdown voltage varied linearly with a ratio of $+20 \mathrm{mV}$ per ${ }^{\circ} \mathrm{C}$. All GAPD measurements with a specific excess bias $\mathrm{V}_{\mathrm{eb}}$ are compensated for changes in $\mathrm{V}_{\mathrm{bd}}$.

At an excess bias of $2.4 \mathrm{~V}$, a one second acquisition of the pill-shaped GAPD's dark count rate (DCR) was $680 \mathrm{~Hz}$ while under a magnetic field of $<0.1 \mathrm{~T}$ and $660 \mathrm{~Hz}$ while under a 9.4T field. The DCR shift was not statistically significant given the inherent shot noise in the measurement. The DCR was observed to double for every increase of roughly $10^{\circ} \mathrm{C}$ ranging from $-20^{\circ} \mathrm{C}$ to $50^{\circ} \mathrm{C}$.

When measuring the rise time of a diode's quench, a $\mathrm{V}_{\mathrm{eb}}$ of $2.4 \mathrm{~V}$, a low threshold of $0.12 \mathrm{~V}\left(5 \%\right.$ of $\left.\mathrm{V}_{\mathrm{eb}}\right)$, and a high threshold of $2.0 \mathrm{~V}$ (roughly $85 \%$ of $\mathrm{V}_{\mathrm{eb}}$ ) were used. The rise time of the diode's quenching waveform, shown for differing magnetic fields in Fig. 4(b), has no significant difference at either of the tested magnetic field orientations. This is evidence that the avalanche propagation speed is the same under a $<0.1 \mathrm{~T}$ or a $9.4 \mathrm{~T}$ field. However, the rise time does vary by $60 \mathrm{ps}$ for temperatures between $-50^{\circ} \mathrm{C}$ and $50^{\circ} \mathrm{C}$, as shown in Fig. 4(c). The rise time variation still includes the variation due to the comparators, as that effect cannot be analyzed separately in this design. Simulations imply this variation is $<10 \mathrm{ps}$ between $-50^{\circ} \mathrm{C}$ and $50^{\circ} \mathrm{C}$.

Using a 34ps, 405nm, pulsed laser, a $12 \mu \mathrm{m}$ GAPD's jitter was acquired in different environments. An optical fiber and mirrors were used to guide the laser beam into and within a temperature chamber or an MRI chamber. The laser was attenuated such that a pulse triggered an avalanche with probability $<1 \%$. A $\mathrm{V}_{\mathrm{eb}}$ of $2.4 \mathrm{~V}$ and a threshold of $0.12 \mathrm{~V}$ were used during the experiment. The resulting jitter, shown in Fig. 3, has a FWHM value of 144ps in a $<0.1 \mathrm{~T}$ field and $152 \mathrm{ps}$ in a 9.4T field. The increase is not significant given that the data is uncompensated for the TDC's DNL.

Overall, measurements imply that avalanche propagation is far more sensitive to changes in temperature than changes in magnetic field. Despite the Lorentz force exerted on the avalanche carriers by a 9.4T field, no effect on propagation speed was observed.

The authors would like to acknowledge Florian Stuker, Katerina Dikaiou, and Yuki Maruyama for their help with the present work, and thank Xilinx, Inc. and Acam Messelectronic GmbH for their donations of hardware.

[1] M. S. Judenhofer et al., "Simultaneous PET-MRI: a new approach for functional and morphological imaging," Nature Medicine, vol. 14, pp. 459-465, 2008. [2] B. J. Pichler et al., "Performance test of an LSO-APD detector in a 7-T MRI scanner for simultaneous PET/MRI," J Nucl Med, vol. 47, no. 4, pp. 639-647, 2006. [3] S. España et al., "Performance evaluation of SiPM photodetectors for PET imaging in the presence of magnetic fields," Nuclear Instruments and Methods in Physics Research Section A: Accelerators, Spectrometers, Detectors and Associated Equipment, vol. 613, no. 2, pp. 308-316, 2010. [4] G. Ripamonti et al., “(PS)2: a new semiconductor device for positron-sensitive picosecond detection of single optical photons," Nuclear Instruments and Methods in Physics Research Section A: Accelerators, Spectrometers, Detectors and Associated Equipment, vol. 310, no. 1-2, pp. 184-188, 1991. [5] B. Swann and other, "A 100-ps time-resolution CMOS time-to-digital converter for positron emission tomography imaging applications," Solid-State Circuits, IEEE Journal of, vol. 39, pp. 1839-1852, Nov. 2004. 


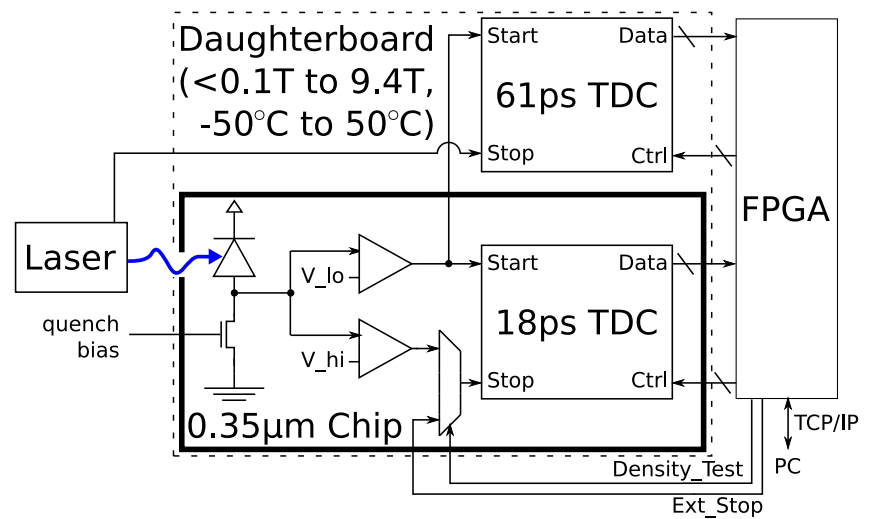

Figure 1: Experimental setup
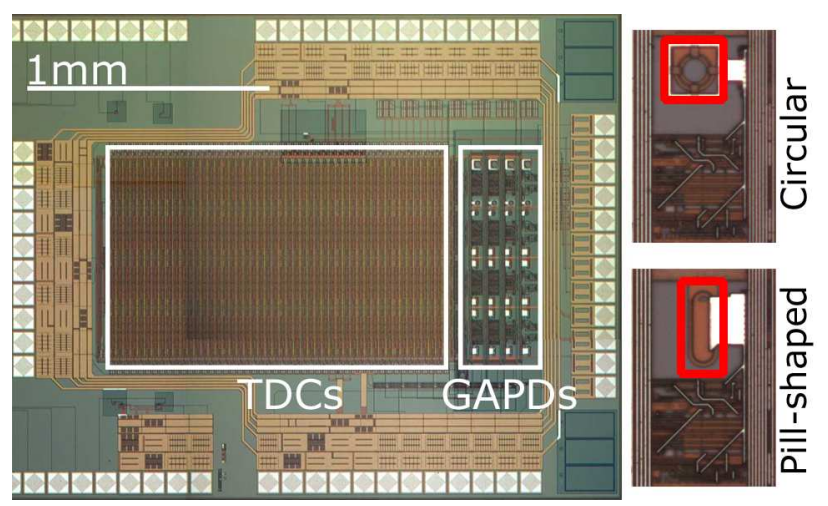

Figure 2: Chip micrograph with enlarged pixels (devices are highlighted in red)

\begin{tabular}{|c|c|c|}
\hline & Property & Value \\
\hline \multirow{4}{*}{ 宅 } & $\mathrm{V}_{\mathrm{bd}}$ of pill-shaped GAPD & $18.6 \mathrm{~V}$ \\
\hline & $\mathrm{V}_{\mathrm{bd}}$ of $12 \mu \mathrm{m}$ GAPD & $18.3 \mathrm{~V}$ \\
\hline & $\mathrm{V}_{\mathrm{bd}}$ temp. dependence & $+20 \mathrm{mV}$ per ${ }^{\circ} \mathrm{C}$ \\
\hline & Dark count rate & $<1 \mathrm{kHz}$ \\
\hline \multirow{6}{*}{ 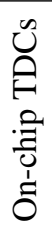 } & Resolution at $<0.1 \mathrm{~T} / 9.4 \mathrm{~T}$ & $18.0 \mathrm{ps} / 18.4 \mathrm{ps}$ \\
\hline & $2 \sigma$ resolution error at $<0.1 \mathrm{~T} / 9.4 \mathrm{~T}$ & $0.1 \mathrm{ps} / 0.5 \mathrm{ps}$ \\
\hline & DNL std. dev. & $0.3 \mathrm{LSB}$ \\
\hline & worst $|\mathrm{DNL}|$ & $<1.1 \mathrm{LSB}$ \\
\hline & worst $|\mathrm{INL}|$ & $<2.0 \mathrm{LSB}$ \\
\hline & TDC jitter & $<5 \mathrm{ps}$ \\
\hline
\end{tabular}

Table 1: Summary table - all results are at room temperature

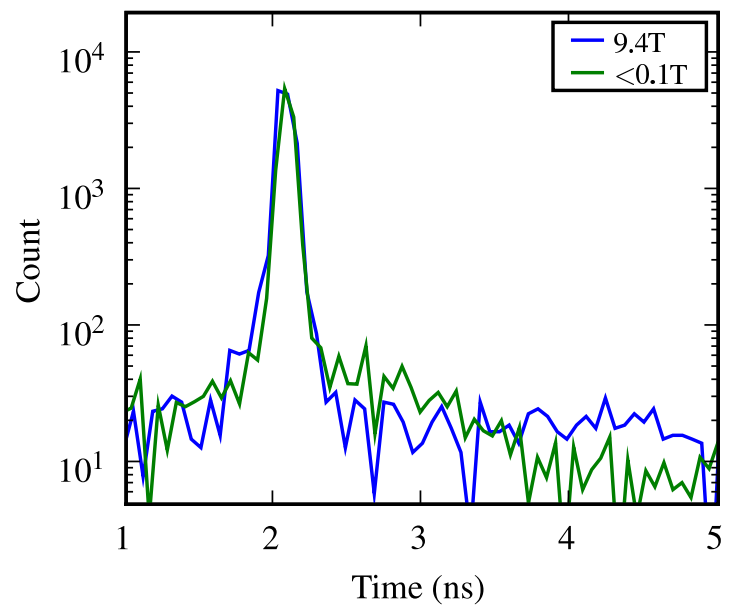

Figure 3: GAPD jitter dependence on environment - shown are jitter measurements of a circular, planar, $12 \mu \mathrm{m}$ GAPD under different magnetic fields. Delay measurements (x-axis) have been normalized so the peaks overlap.

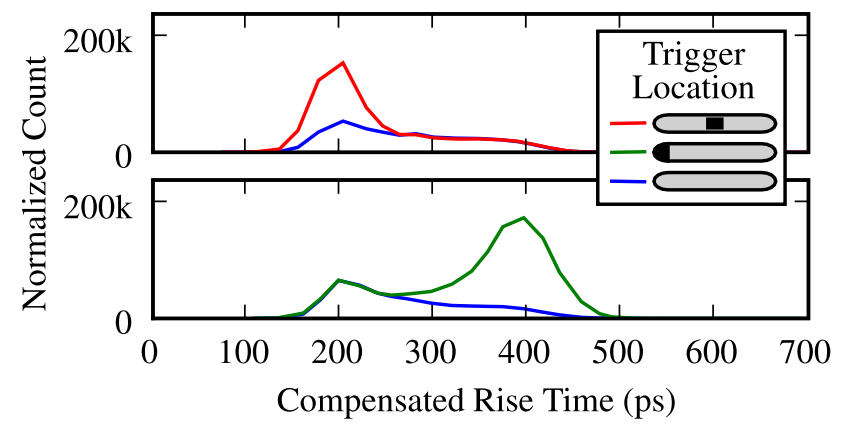

(a) Position sensitivity

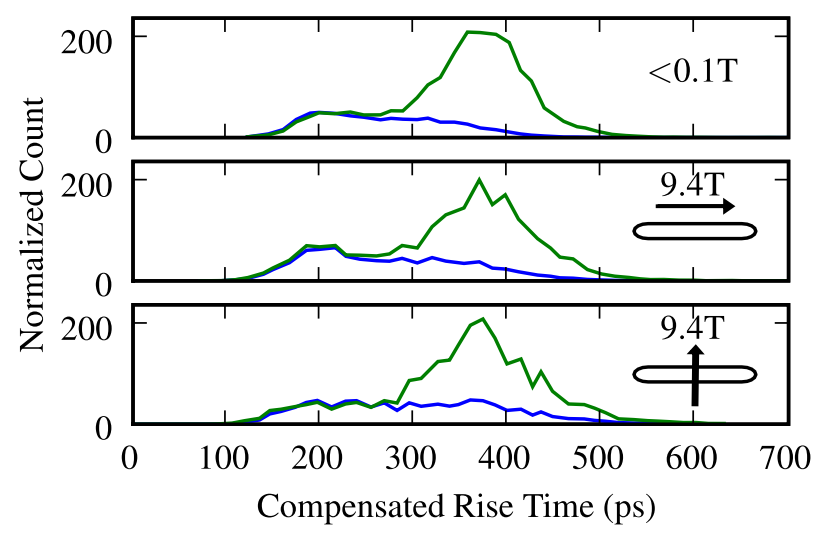

(b) B-field dependence

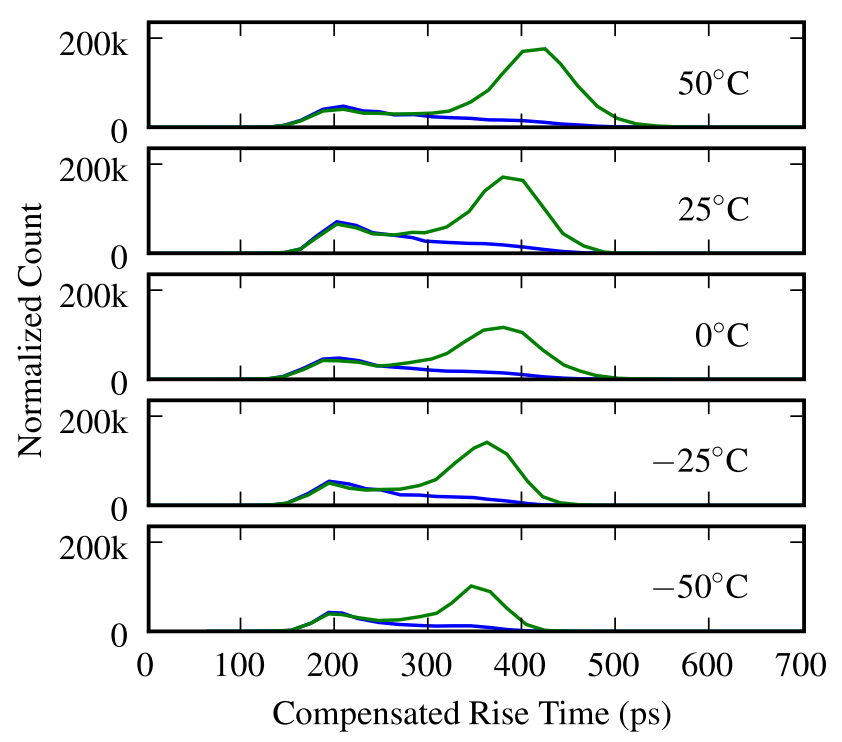

(c) Temperature

Figure 4: Quench times - (a) shows how the sampled rise time of the quench waveform varies in a long but thin diode for predominantly center-seeded avalanches and edge-seeded avalanches compared to avalanches caused by dark counts. Recovering the rise time of center-seeded avalanches using the distribution in the dark, (b) and (c) show how the quench time depends on environmental conditions. The 18ps TDC measured the quench time while the diodes had the following operating conditions: $\mathrm{V}_{\mathrm{eb}}$ of $2.4 \mathrm{~V}$, low threshold of $0.12 \mathrm{~V}$, and high threshold of $2.0 \mathrm{~V}$. Both the $\mathrm{x}$ - and $\mathrm{y}$-axis are compensated for nonuniformities in the TDCs' DNLs, and the $\mathrm{x}$-axis has been shifted to align the peak from avalanches caused by dark counts. 